TITLE:

\title{
Modeling configurational energetics on multiple lattices through extended cluster expansion
}

$\operatorname{AUTHOR}(S)$ :

Yuge, Koretaka

CITATION:

Yuge, Koretaka. Modeling configurational energetics on multiple

lattices through extended cluster expansion. Physical Review B 2012, 85(14): 144105.

ISSUE DATE:

2012-04-09

URL:

http://hdl.handle.net/2433/188005

RIGHT:

(C)2012 American Physical Society 
PHYSICAL REVIEW B 85, 144105 (2012)

\title{
Modeling configurational energetics on multiple lattices through extended cluster expansion
}

\author{
Koretaka Yuge \\ Department of Materials Science and Engineering, Kyoto University, Sakyo, Kyoto 606-8501, Japan \\ (Received 27 November 2011; revised manuscript received 21 March 2012; published 9 April 2012)
}

\begin{abstract}
We demonstrate the applicability of an extended cluster expansion (CE) technique, variable-lattice CE (VLCE), enabling configurational energetics on multiple lattices to be modeled. For application in real systems, the general conditions of determining interactions in VLCE is derived. As an example, VLCE was applied to a boron nitride (BN) binary system on two-dimensional lattices. VLCE successfully predicted the energy of structures, not only on lattices that are used to obtain interactions, but also of structures on those not used to obtain interactions. VLCE reasonably predicts the stable structure of $\mathrm{BN}$ on a honeycomb lattice from information about other lattices. These facts indicate that VLCE opens the door to effectively searching for stable as well as metastable structures on multiple lattices for unknown given systems, which has not been achieved by the current CE.
\end{abstract}

DOI: 10.1103/PhysRevB.85.144105

PACS number(s): 61.50.Ah, 64.60.-i, 81.30.-t

\section{INTRODUCTION}

Structures of alloys, including atomic arrangements and compositions, should significantly affect physical properties. Knowing the alloy configurational thermodynamics as well as the relationship between the property and structure is thus a fundamental prerequisite for the design of suitable alloy materials in terms of narrowing down the controlling parameters. However, assessing alloy thermodynamics or capturing characteristic properties in configuration space requires a tremendous number of atomic arrangements, which is typically far beyond the practical limitation of density functional theory (DFT) calculations. Alternative approaches therefore have been proposed to reduce the computational cost of DFT. The cluster expansion (CE) technique ${ }^{1,2}$ is one of the most promising and well-established approaches to give an accurate prediction of configuration-dependent scalar properties. CE combined with DFT has been applied to a wide variety of systems, including phase stability for binary $^{3-5}$ and multicomponent ${ }^{6-8}$ bulk alloys, surface ordering and segregation, ${ }^{9-12}$ alloy nanoparticles, ${ }^{13}$ effects of lattice vibration $^{14,15}$ and of external pressure ${ }^{16}$ on phase stability, electronic density of states for disordered alloys, ${ }^{17}$ and the comprehensive search for superhard materials. ${ }^{18}$ The form of the $\mathrm{CE}$ has been modified in various manners for systems that require specific treatments in terms of configuration spaces: mixed-space $\mathrm{CE}^{19,20}$ for long-period superlattices, coupled $\mathrm{CE}^{21}$ for adsorption-induced surface segregation and ionic systems, and tensorial $\mathrm{CE}^{22}$ enables the treatment of tensorvalued properties, such as elasticity and dielectric constants. Another modification of the CE is to increase the accuracy of the predicted values by optimizing selected clusters as well as DFT input structures to obtain effective interactions. ${ }^{23-25}$ Thus, CE has become a powerful tool to theoretically optimize alloy materials in terms of atomic arrangements.

In spite of such successful modifications, the applicability of the current $\mathrm{CE}$ is essentially confined to a given single lattice, indicating that $\mathrm{CE}$ can miss stable or metastable structures and their properties on lattices which are not attributed to the lattice where the $\mathrm{CE}$ interactions are constructed. Therefore, in order to achieve a general design of alloy materials beyond a given single lattice, it is natural that $\mathrm{CE}$ should require modification to treat multiple lattices. Very recently, the author has developed extended CE, variable-lattice CE (VLCE) ${ }^{26}$ to overcome the essential limitation of CE. In VLCE, interactions are independent of atomic arrangements as well as lattices, which means that VLCE can handle multiple lattices simultaneously. However, in VLCE, due to the use of an abstract lattice to describe the positions of lattice points in multiple lattices, conventional classification of interactions according to the symmetry of the system, which is a central advantage of $\mathrm{CE}$, cannot be applied, which has prevented the practical application of VLCE to real systems so far. In the present study, we successfully found the general conditions for the classification of VLCE interactions which enables its application to real systems. We use the example of searching stable atomic arrangements on multiple lattices for a boron nitride $(\mathrm{BN})$ two-dimensional system based on the combination of VLCE and DFT, which certainly indicates that VLCE can effectively handle configurational energetics on multiple lattices.

\section{METHODOLOGY}

We first briefly introduce the concept and form of VLCE, since the detailed derivation and concept of VLCE are described in Ref. 26. In VLCE, multiple lattices are linked via a combination of two lattices: base and virtual lattices. The former specifies the occupation of elements on a certain given lattice, which is similar to conventional CE. The latter specifies the position of individual lattice points or a set of lattice points measured from the base lattice. Therefore, the base lattice is a real lattice, while the virtual lattice is merely an abstract lattice that has no concrete concept of symmetry. The basis function for base and virtual lattices can be constructed by a standard orthonormalization technique used in CE, and taking the tensor product of vector space for base and virtual lattices leads to a general VLCE expression for energy $E$ :

$$
\begin{aligned}
E(\vec{\sigma}, \vec{\tau}) & =\sum_{\alpha, \beta} \sum_{(M, L)} \Phi_{\alpha ; \beta}^{(M, L)}(\vec{\sigma}, \vec{\tau}) V_{\alpha ; \beta}^{(M, L)}, \\
\Phi_{\alpha ; \beta}^{(M, L)}(\vec{\sigma}, \vec{\tau}) & =\prod_{\substack{i \in \alpha \\
d \in(M)}} \phi_{d}\left(\sigma_{i}\right) \prod_{\substack{p \in \beta \\
d^{\prime} \in(L)}} \phi_{d^{\prime}}\left(\tau_{p}\right),
\end{aligned}
$$


where $\Phi_{\alpha ; \beta}^{(M, L)}(\vec{\sigma}, \vec{\tau})$ is an expansion function, and is called a cluster function. $\alpha$ and $\beta$ represent clusters consisting of lattice points $\{i\}$ and $\{p\}$ on base and virtual lattices, and $(M)$ and $(L)$ specify the combination of basis function index $d$ and $d^{\prime}$ on base and virtual lattices, respectively. $\sigma$ and $\tau$ are spin variables defined on base and virtual lattices, and individually have integer values to specify the occupation of elements or the position of lattice points, which are similar to spin variables in the Ising model. $V_{\alpha ; \beta}^{(M, L)}$ is an expansion coefficient and is called a VLCE effective cluster interaction (ECI). ECIs are defined on base, virtual, and coupling between base and virtual lattices. In order to obtain ECIs, DFT energies for several structures are typically least-squares fitted to Eq. (1). Due to the limitation of the number of DFT energies, Eq. (1) should be terminated at finite order. To achieve practical termination, one of the greatest advantages of conventional $\mathrm{CE}$ is that the number of clusters can be significantly reduced [i.e., reduce the number of expansion terms in Eq. (1)] according to the symmetry of a given lattice. In VLCE, since we treat multiple lattices with different symmetry and the virtual lattice is an abstract lattice, cluster classification is not straightforward. Let us consider a system with $r$ symmetry-nonequivalent structures on $q$ lattices, where the system has totally $N$ structures on $q$ lattices when all symmetry-equivalent structures are explicitly taken into account. The VLCE-independent clusters are considered to be a minimal complete set of basis to describe the $r$ structures with their corresponding cluster functions, which therefore can be determined through the following equation:

$$
\left(\Phi_{\text {aug }}\right)^{-1}\left(\hat{T}_{\text {aug }} \cdot \mathbf{f}\right)=\hat{U}_{\text {aug }} \cdot \mathbf{V}_{\mathrm{VLCE}},
$$

where $\Phi_{\text {aug }}$ is an $N \times N$ cluster function matrix consisting of all $N$ structures and clusters derived from symmetry operation in $r$ structures on $q$ lattices. $\mathbf{f}$ is an $r$-component vector of property $f$ for $r$ structures, and operator $\hat{T}_{\text {aug }}$ transforms $\mathbf{f}$ into an $N$-component vector according to the symmetry of $r$ structures so that each component corresponds to $\Phi_{\text {aug }} . \mathbf{V}_{\mathrm{VLCE}}$ is composed of ECIs for VLCE-independent clusters, and $\hat{U}_{\text {aug }}$ is a similar operator to $\hat{T}_{\text {aug }}$, which transforms $\mathbf{V}_{\mathrm{VLCE}}$ into an $N$ component vector. For instance, the element of matrix $\Phi_{\text {aug }}$ is

$$
\Phi_{r s}=\Phi_{s}\left(\vec{\sigma}_{r}, \vec{\tau}_{r}\right),
$$

where $s$ specifies the cluster type and basis function index [i.e., $\alpha, \beta$, and $(M, L)$ in Eq. (1)]. The vector $\mathbf{f}$ is composed of $r$ values of $f_{1}, f_{2}, \ldots, f_{r}$ and $\hat{T}_{\text {aug }} \mathbf{f}$ has $N$ components, each of which should take one of the $r$ values of the component in f. When structure $g$ has value $f_{g}$ in vector $\mathbf{f}$, all the structures that are symmetry-equivalent to the structure $g$ take the same value of $f_{g}$ in $\hat{T}_{\text {aug }} \mathbf{f}$. The vector $\mathbf{V}_{\mathrm{VLCE}}$ is not known in advance, while $\hat{U}_{\text {aug }} \mathbf{V}_{\mathrm{VLCE}}$ has $N$ components of $v_{1}, v_{2}, \ldots, v_{N}$, each of which corresponds to matrix elements $\Phi_{\text {aug }}$ [i.e., $v_{k}$ to $\Phi_{k}$ in Eq. (3)]. Each component of $\hat{U}_{\text {aug }} \mathbf{V}_{\mathrm{VLCE}}$ corresponds to VLCE-ECI when all equivalent VLCE clusters are explicitly distinguished. Therefore, in Eq. (2), information of symmetry for multiple lattices is automatically included to classify VLCE-independent clusters instead of classifying clusters according to the symmetry of a single empty lattice in conventional CE. Since $\Phi_{\text {aug }}$ can be directly estimated for given $r$ structures by Eqs. (1) and (3) when all possible clusters are explicitly distinguished, and $\hat{T}_{\text {aug }}$ is obtained through

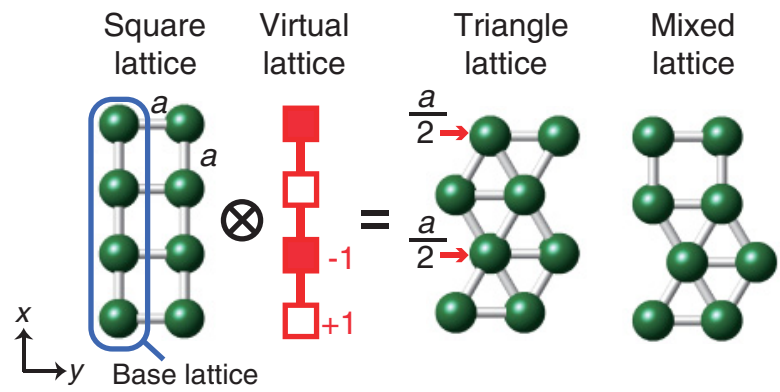

FIG. 1. (Color online) Schematic illustration of how multiple lattices are derived from coupling of the base lattice (here, square lattice) and virtual lattice.

symmetry operation of $r$ structures described above, VLCEindependent clusters can be determined just by substituting several sets of artificially prepared $\mathbf{f}$ into the left-hand side of Eq. (2) and numerically finding the characteristics of operator $\hat{U}_{\text {aug }}$ where the certain VLCE-independent ECI is transformed into equivalent ECIs, treating all possible $N$ clusters explicitly.

In order to confirm the applicability of the VLCE based on Eq. (2) to a real system, we assessed the energetics for the BN binary system on multiple two-dimensional lattices, where the coupling contribution between atomic arrangements and lattice types is considered to be significant. Since three dimensionally, $\mathrm{BN}$ prefers a hexagonal lattice, the constituent honeycomb lattice is expected to be one of the energetically favored lattices in two dimensions. In the present work, we chose other lattices for DFT input structures in order to confirm the predictive power of VLCE for stable structures. First, a square lattice with four sites and with the lattice parameter of $a$ and $4 a$ was chosen as the base lattice, as shown on the left-hand side of Fig. 1. Spin variable $\sigma$ on the base lattice was defined as $\sigma_{i}=+1(-1)$, specifying the occupation of the $\mathrm{B}(\mathrm{N})$ atom at lattice point $i$. Next, a virtual lattice was defined where each lattice point specifies the position of the base lattice points with the same $x$ positions. Here, $\tau$ on the virtual lattice is defined as $\tau=+1$, representing that corresponding lattice points remain in their original position, and $\tau=-1$ represents displacement of base lattice points by $+a / 2$ in the $y$ direction. The schematic role of the defined virtual lattice and resultant derived lattice (here, triangle lattice) is illustrated in the middle of Fig. 1. Combination of base and virtual lattices thus can produce a number of atomic arrangements on multiple lattices. Since $\sigma$ and $\tau$, respectively, have two values of \pm 1 , the VLCE expression of energy in Eq. (1) is significantly simplified as

$$
E(\vec{\sigma}, \vec{\tau})=V_{0}+\sum_{\alpha, \beta}\left(\left\langle\prod_{i \in \alpha} \sigma_{i} \prod_{p \in \beta} \tau_{p}\right\rangle V_{\alpha ; \beta}\right),
$$

where the summation is taken over VLCE-independent clusters $\{\alpha ; \beta\}$ determined by Eq. (2), and the bracket \langle\rangle denotes the average over all clusters equivalent to class $(\alpha ; \beta)$. In order to obtain the ECIs of $V_{\alpha ; \beta}$, we constructed ordered structures on three types of lattices consisting of square, triangle, and a mixture of square and triangle lattices, as shown in Fig. 1, for DFT input structures. A unit cell with four atoms defined on the base lattice is illustrated on the left-hand side of Fig. 1. Within the four-atom 


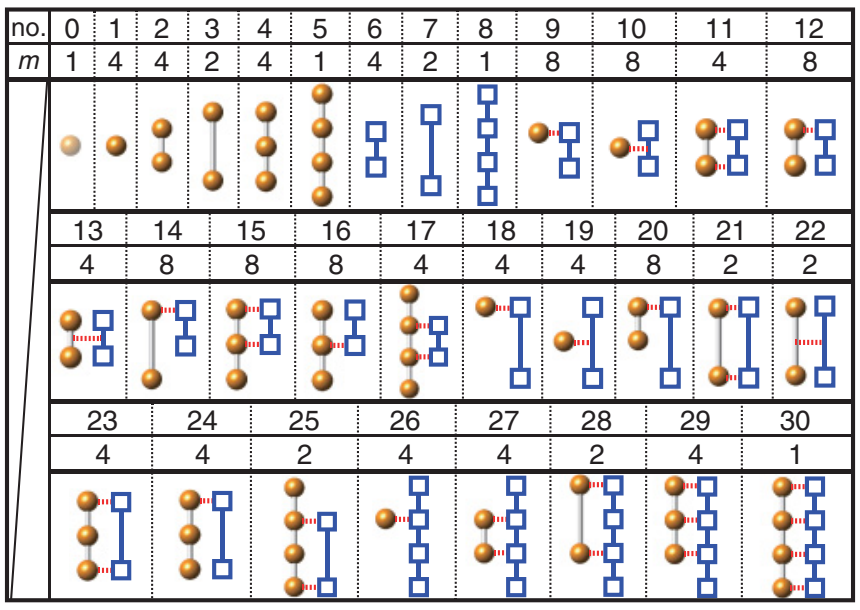

FIG. 2. (Color online) VLCE-independent clusters obtained from Eq. (2). Cluster number (no.) and the number of clusters in the unit cell $(m)$ are described together. Filled spheres denote base lattice points and open squares denote virtual lattice points. Lattice points connected with dotted lines represent that they correspond to the same lattice points on a well-defined lattice.

cells, there are 31 symmetry-nonequivalent structures derived from the base and virtual lattices. For these structures, the operator $\hat{T}_{\text {aug }}$ transforms f with 31 components into that with 256 components. Applying this operator to Eq. (2), we obtain 31 VLCE-independent clusters, shown in Fig. 2. Cluster number and the number of clusters in unit cell $(\mathrm{m})$ are described together. Clusters with spheres are on the base lattice, those with squares are on the virtual lattice, and others are coupled between the base and virtual lattices, respectively.

We employ first-principles calculations using a DFT code, the Vienna $a b$ initio simulation Package (VASP), ${ }^{27,28}$ to obtain total energies for ordered structures in $\mathrm{BN}$ alloys, which are least-squares fitted to the VLCE Hamiltonian in Eq. (4). We estimate the electronic contribution to the total energy for 17 ordered structures on the three lattices. All-electron Kohn-Sham equations are solved by employing the projector augmented-wave (PAW) method, ${ }^{29,30}$ within the generalized gradient approximation (GGA) of Perdew-Wang 91 form, ${ }^{31}$ to an exchange-correlation functional. Plane-wave cutoff energy is set at $500 \mathrm{eV}$. For the 17 structures, initial internal atomic positions are defined by the base and virtual lattices shown in Fig. 1. Initial cell size is defined as $a=2.0 \AA$ in Fig. 1 for $x$ and $y$ directions, and cell size in the $z$ direction is fixed at $16 \AA$ to avoid interactions between neighboring two-dimensional structures. Cell size in $x$ and $y$ directions are independently changed to find local minima in energy that is closest to the initial cell, where internal atomic positions are optimized with residual forces less than $0.01 \mathrm{eV} / \AA$ for each size of cell. This procedure indicates that optimized structure can be locally (or globally) stable, which is closest to the initial state defined by base and virtual lattices. Note that the strain of the interface between different lattice types would be automatically included in the VLCE-ECI by applying the energies for the optimized structures, while the strain of the interface, where the constituent lattice has a long period, is expected to be difficult to efficiently handle with the current VLCE for reasons similar to the CE for treating long-period lattice-mismatched superlattices. ${ }^{19}$ In order to modify this, explicit inclusion of elastic strain energy, which has been suggested in mixed-space $\mathrm{CE},{ }^{19}$ to VLCE formalism would be needed, while the inclusion of such strain energy is out of the present scope. Brillouin zone integration is performed on the basis of the Monkhorst-Pack scheme ${ }^{32}$ with a $2 \times 8 \times 1$ $k$-point mesh in terms of the initial unit cell of $8 \times 2 \times 16 \AA$ for a square lattice. Note that the 17 structures are chosen among possible structures on the three lattices so that they can successfully retain a two-dimensional structure.

\section{RESULTS AND DISCUSSION}

Applying the energies of 17 structures to a VLCE Hamiltonian, we obtain a set of 12 optimal VLCE multibody clusters and ECIs determined according to our previous studies, ${ }^{33,34}$ employing a genetic algorism ${ }^{35}$ to minimize the cross-validation (CV) score. ${ }^{36}$ In the present study, the CV score takes $0.063 \mathrm{eV} /$ site, where the standard deviation of the DFT energy for the 17 input structures is $0.7 \mathrm{eV} /$ site. Resultant ECIs for the multibody clusters are shown in Fig. 3. The 12 clusters consist of 3, 2, and 7 clusters on base, virtual, and coupling lattices, respectively. Cluster no. 2 [i.e., first nearest-neighbor (1-NN)] has the largest magnitude with a positive sign, indicating that $\mathrm{BN}$ prefers an unlike-atom pair in nearest-neighbor coordination. Using the ECIs in Fig. 3, we can now estimate energies for ordered structures on lattices other than square, triangle, or mixed lattices. In order to see the predictive power of the current VLCE-ECIs, we compare energies predicted by DFT and VLCE, as shown in Fig. 4. The left part of Fig. 4 (i.e., "Included") corresponds to the 17 ordered structures that are used to obtain ECIs in Fig. 3, and the right part (i.e., "Not included") corresponds to structures on lattices that are not used to obtain ECIs. We can clearly see that VLCE reasonably predicts energies for the 17 DFT input structures on multiple lattices. A more important result is presented in the right part of Fig. 4: VLCE can successfully predict energies within an error of $\mathrm{CV}$ score for structures on honeycomb lattices and more complicated lattices that are not used to construct ECIs. Here, six structures in the right part

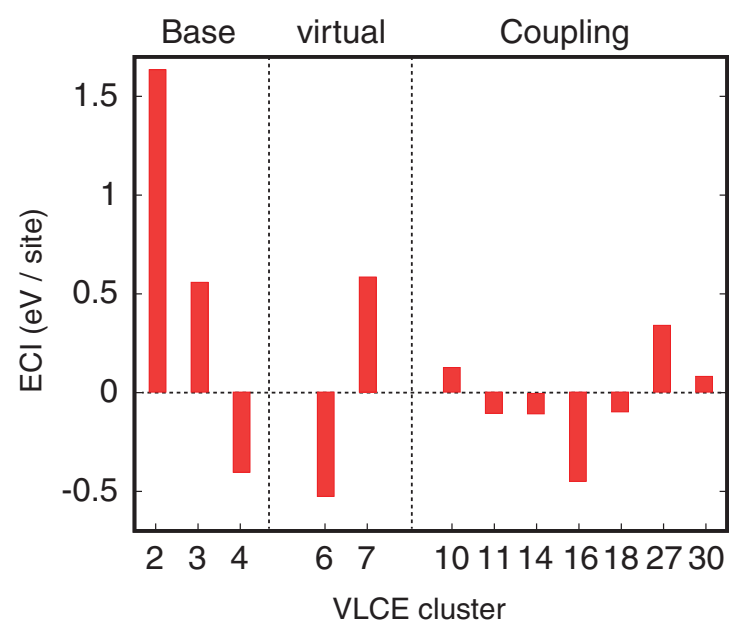

FIG. 3. (Color online) VLCE effective cluster interactions for an optimal set of 12 multibody clusters. 


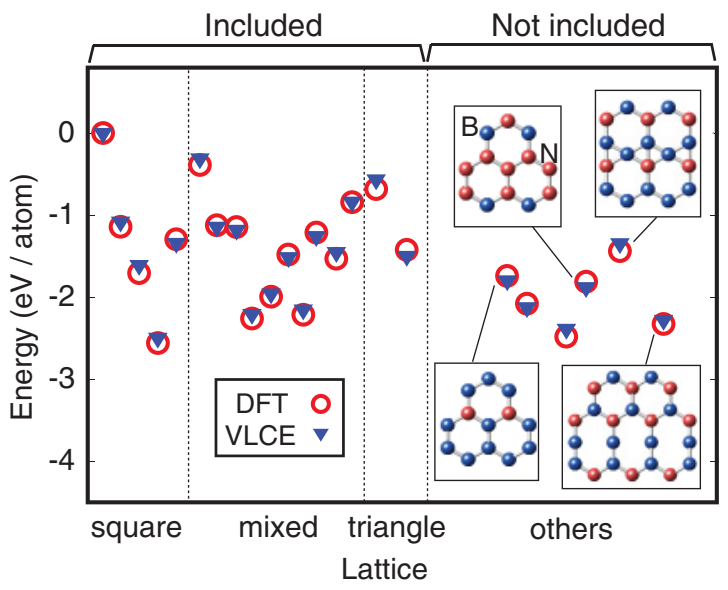

FIG. 4. (Color online) Comparison of energies for multiple lattices predicted by DFT (open circles) and VLCE (filled triangles). "Included" denotes structures that are used to obtain ECIs in Fig. 3, and "Not included" denotes those on other lattices that are not used to obtain ECIs.

consist of 3-5 atoms on different lattices and were found to successfully retain a two-dimensional structure.

Using the ECIs in Fig. 3, we can also effectively search stable as well as metastable atomic arrangements on a variety of lattices that are derived from the defined base and virtual lattices. In order to assess stable structures, we perform Monte Carlo (MC) simulation with a simulated annealing algorism under the Metropolis algorism for the BN binary system with 128 atoms at equiatomic composition. Three thousand MC steps per site are employed and, at each step, total energy in the system is recorded. Figure 5 shows the resultant energies as a function of MC steps. Most stable structures and some other structures with a small number of atoms in cells are illustrated, and VLCE-predicted energy measured from DFT energy in units of $(\mathrm{eV} / \mathrm{site})$ is described for each structure. The difference in energy reasonably ranges around the order of the CV score. The present VLCE-ECI predicts that the most stable structure is monolayer h-BN, shown on the rightmost side of the structure in Fig. 5, which is expected to be one of the stable structures for the two-dimensional BN system. In the above discussions, the VLCE-ECIs are constructed based on ordered structures on three lattices of square, triangle, and their mixed lattices that are expected not to be very stable; the ECIs do not have information about stable structures on stable lattices, as shown in Fig. 5. By applying the similar MC simulation in Fig. 5 to other compositions of $x=0.25$ and 0.75 ( $x$ is defined as $\mathrm{B}_{x} \mathrm{~N}_{(1-x)}$ ), we obtain 12 stable and metastable structures consisting of up to eight atoms. When we add DFT energies for the 12 structures to obtain the VLCE-ECI, the resultant $\mathrm{CV}$ score is significantly reduced from 0.063 to $0.012 \mathrm{eV} /$ site, which can be partially attributed to the smaller number of initial input structures of 17 . This finding therefore indicates that the predictive power of VLCE for structures

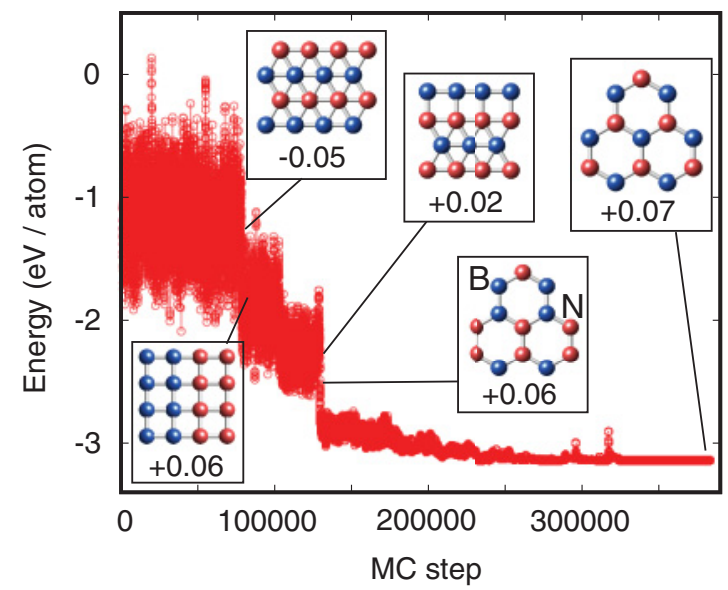

FIG. 5. (Color online) VLCE-predicted energy for atomic arrangements on a number of lattices as a function of $\mathrm{MC}$ step obtained through MC simulation with simulated annealing algorism. Predicted energy measured from DFT energy is described together with ordered structures.

on multiple lattices can be reasonably reduced by adding an appropriate set of structures in a similar fashion to the manner developed in conventional CE.

\section{CONCLUSION}

We have demonstrated that VLCE opens the door for predicting configurational energetics on multiple lattices, which is a potential tool for systems where the lattice type of stable as well as metastable structures are not experimentally well known. We develop a cluster classification method that is specific to the VLCE, which enables accurate prediction of energy on multiple lattices. We find that predictive power of VLCE can be reasonably modified by adding an appropriate set of structures in a similar fashion to conventional CE. VLCE is particularly effective for systems that can be described by a combination of common partial lattices, which holds for the present case of BN systems. For other systems, especially with a different number of atoms in the cell, another extended $\mathrm{CE}$ approach of grid-increment $\mathrm{CE}$ (GICE) ${ }^{37}$ that we recently developed would be more efficient to treat such multiple lattices; however, since the virtual lattice is an abstract lattice, it can be coupled with any type of real lattice, including the lattices used in the GICE. The combination of VLCE and GICE is expected to enable a more global and comprehensive search of properties in terms of all possible atomic arrangements on multiple lattices, which should be developed in a future study.

\section{ACKNOWLEDGMENTS}

This study was supported by a Grant-in-Aid for Young Scientists B (22760502) from JSPS.
${ }^{1}$ J. M. Sanchez, F. Ducastelle, and D. Gratias, Physica A 128, 334 (1984).

${ }^{2}$ J. M. Sanchez and J. D. Becker, Mater. Res. Soc. Symp. Proc. 291, 115 (1993).
${ }^{3}$ Z. W. Lu, S.-H. Wei, and A. Zunger, Phys. Rev. Lett. 66, 1753 (1991).

${ }^{4}$ M. Asta, D. de Fontaine, M. van Schilfgaarde, M. Sluiter, and M. Methfessel, Phys. Rev. B 46, 5055 (1992). 
${ }^{5}$ A. van de Walle and G. Ceder, J. Phase Equilib. 23, 348 (2002).

${ }^{6}$ C. Wolverton and D. de Fontaine, Phys. Rev. B 49, 8627 (1994).

${ }^{7}$ F. Lechermann, M. Fähnle, and J. M. Sanchez, Intermetallics 13, 1096 (2005).

${ }^{8}$ K. Yuge, A. Seko, Y. Koyama, F. Oba, and I. Tanaka, Phys. Rev. B 77, 094121 (2008).

${ }^{9}$ A. V. Ruban and H. L. Skriver, Comput. Mater. Sci. 15, 119 (1999).

${ }^{10}$ R. Drautz, H. Reichert, M. Fähnle, H. Dosch, and J. M. Sanchez, Phys. Rev. Lett. 87, 236102 (2001).

${ }^{11}$ K. Yuge, A. Seko, A. Kuwabara, F. Oba, and I. Tanaka, Phys. Rev. B 74, 174202 (2006).

${ }^{12}$ S. Müller, M. Stöhr, and O. Wieckhorst, Appl. Phys. A 82, 415 (2006).

${ }^{13}$ K. Yuge, Phys. Rev. B 84, 085451 (2011).

${ }^{14}$ G. D. Garbulsky and G. Ceder, Phys. Rev. B 53, 8993 (1996).

${ }^{15}$ A. van de Walle and G. Ceder, Rev. Mod. Phys. 74, 11 (2002).

${ }^{16}$ K. Yuge, J. Phys.: Condens. Matter. 21, 055403 (2009).

${ }^{17}$ H. Y. Geng, M. H. F. Sluiter, and N. X. Chen, J. Chem. Phys. 122, 214706 (2005).

${ }^{18}$ K. Yuge, J. Phys.: Condens. Matter 21, 415403 (2009).

${ }^{19}$ D. B. Laks, L. G. Ferreira, S. Froyen, and A. Zunger, Phys. Rev. B 46, 12587 (1992).
${ }^{20}$ V. Ozoliņš, C. Wolverton, and A. Zunger, Phys. Rev. B 57, 6427 (1998).

${ }^{21}$ B. C. Han, A. Van der Ven, G. Ceder, and B. J. Hwang, Phys. Rev. B 72, 205409 (2005).

${ }^{22}$ A. van de Walle, Nature Mater. 7, 455 (2008).

${ }^{23}$ V. Blum, G. L. W. Hart, M. J. Walorski, and A. Zunger, Phys. Rev. B 72, 165113 (2005).

${ }^{24}$ A. Seko, Y. Koyama, and I. Tanaka, Phys. Rev. B 80, 165122 (2009).

${ }^{25}$ T. Mueller and G. Ceder, Phys. Rev. B 82, 184107 (2010).

${ }^{26}$ K. Yuge, J. Phys.: Condens. Matter 22, 125402 (2010).

${ }^{27}$ G. Kresse and J. Hafner, Phys. Rev. B 47, R558 (1993).

${ }^{28}$ G. Kresse and J. Furthmüller, Phys. Rev. B 54, 11169 (1996).

${ }^{29}$ G. Kresse and D. Joubert, Phys. Rev. B 59, 1758 (1999).

${ }^{30}$ P. E. Blöchl, Phys. Rev. B 50, 17953 (1994).

${ }^{31}$ J. P. Perdew and Y. Wang, Phys. Rev. B 45, 13244 (1992).

${ }^{32}$ H. J. Monkhost and J. D. Pack, Phys. Rev. B 13, 5188 (1976).

${ }^{33}$ K. Yuge, J. Phys.: Condens. Matter 21, 415401 (2009).

${ }^{34}$ K. Yuge, Phys. Rev. B 79, 144109 (2009).

${ }^{35}$ G. L. W. Hart, V. Blum, M. J. Walorski, and A. Zunger, Nature Mater. 4, 391 (2005).

${ }^{36}$ A. van de Walle, M. Asta, and G. Ceder, Calphad 26, 539 (2002).

${ }^{37}$ K. Yuge, Calphad 36, 23 (2012). 